\title{
The effects of meson mixing on dilepton spectra
}

\author{
O. Teodorescu, A. K. Dutt-Mazumder and C. Gale \\ Physics Department, McGill University \\ 3600 University St., Montreal, Quebec H3A 2T8, Canada
}

\begin{abstract}
The effect of scalar and vector meson mixing on the dilepton radiation from hot and dense hadronic matter is estimated in different isospin channels. In particular, we study the effect of $\sigma-\omega$ and $\rho-a_{0}$ mixing and calculate the corresponding rates. Effects are found to be significant compared to standard $\pi-\pi$ and $K-\bar{K}$ annihilations. While the mixing in the isoscalar channel mostly gives a contribution in the invariant mass range between the two-pion threshold and the $\omega$ peak, the isovector channel mixing induces an additional peak just below that of the $\phi$. Experimentally, the dilepton signals from $\rho$ - $a_{0}$ mixing seem to be more tractable than those from $\sigma$ - $\omega$ mixing.
\end{abstract}

PACS numbers: 25.75.-q, 25.75Dw, 24.10Cn

Keywords: quantum hadrodynamics, vector-scalar mixing, dilepton spectra

\section{INTRODUCTION}

There exists physical processes which are forbidden in free space but can take place in matter. Those are basically related to medium-induced symmetry breaking effects. The interaction respects the symmetry, but the ground state breaks it [1] 3]. For instance, in matter, Lorentz symmetry is lost which leads to the mixing of different spin states even when the interaction Lagrangian respects all the required symmetry properties. A well-known example of this is $\sigma-\omega$ mixing in nuclear matter as was first shown by Chin [4] or $\rho$ - $a_{0}$ mixing as pointed out in Ref. [5. In addition there might be mixing between different isospin states in asymmetric nuclear matter. The $\rho-\omega$ mixing provides one such example as suggested in [1].

Our goal here is to identify the effects of scalar and vector meson mixing on dilepton production rates, which could in turn be observed in high energy heavy ion experiments. It is well known that the electromagnetic radiation provides a penetrating probe to study various in-medium properties of vector mesons. Stimulated by the idea that in nuclear matter the vector meson properties might be modified from their vacuum values as a precursor phenomenon to chiral symmetry restoration, a great deal of effort has been directed to understand their properties in hot and/or dense nuclear matter [6,7]. Several experiments have measured, or are planning to measure, dilepton pair production in nucleus-nucleus collisions. Such measurements have been carried out by the Dilepton Spectrometer (DLS) at Lawrence Berkeley National Laboratory [8], and by HELIOS [9] and CERES [10] at CERN. The NA50 collaboration also measures dileptons yields in the light vector meson mass range 11. Two new initiatives to be mentioned in this context are PHENIX 12] at the Relativistic Heavy Ion Collider (RHIC) at Brookhaven National Laboratory and HADES at the Gesellschaft für Schwerionenforschung (GSI) [13]. Density-dependent effects can also be found in experiments at the Thomas Jefferson National Accelerator Facility (TJNAF) [14]. As the effects to be discussed here are mostly density-driven, the latter two facilities represent the energy regime and physical conditions perhaps more relevant for the present study. However, the physics discussed here could also be highlighted in an eventual low-energy RHIC run.

For the understanding of the data in these experiments it is important to uncover the various processes which might lead to the production of dileptons and to estimate their relative contribution to the total production rate. Some effort has already being put in obtaining some "standard" sources related to the decay channels of various light mesons and processes like $\pi-\pi$ or $K-\bar{K}$ annihilation 15 17. But the opening-up of new channels related to scalar-vector mixing, which constitutes the material to be presented in this paper, has only been addressed recently.

The mixing of mesons considered here includes both the isoscalar and isovector channels. To be more specific, we estimate the rate of dilepton production from $\sigma-\omega$ and $\rho-a_{0}$ mixing. Even though some attention was paid to the former in the context of heavy-ion collisions 18.19, the importance of the latter was shown only recently in a zero temperature estimate of the dilepton production cross section [5]. The studies of the effects of mixing made so far were limited to calculations of cross-sections.

This paper reports on calculations of the thermal production rates of dileptons induced by scalar-vector mixing in the isoscalar and isovector channels. Those results are then compared against standard $\pi-\pi, K-\bar{K}$ annihilations, at different temperatures and chemical potentials. Note that processes involving mixing of different G-parity states like $\sigma-\omega$ or $\rho-a_{0}$ are allowed only in matter which is not invariant under charge conjugation. Therefore, $\pi+\pi \rightarrow e^{+} e^{-}$ through their coupling to nucleons via $\sigma$ and $\omega$ or $\pi+\eta \rightarrow e^{+} e^{-}$mediated by $\rho-a_{0}$ mixing, can take place, as will become clear later, only in matter with finite nucleon chemical potential. Hence a natural place to look for such 
density-driven effects are experiments involving relatively low energy where one expects to have a relatively large chemical potential.

The model used here is Walecka-like with the inclusion of the $a_{0}$ meson and its mixing with the $\rho$ meson. The role of $a_{0}$ in the context of nuclear matter calculation has also been discussed in Ref. [5,20 22]. To keep the calculation simple we take nuclear matter to be symmetric under $p \leftrightarrow n$ but this can easily be generalized to the case where nuclear matter has different number of protons and neutrons.

In section II we discuss the general outline of the formalism. Next in section III we discuss $\sigma$ - $\omega$ and $\rho$ - $a_{0}$ mixing. In section IV we present our results for various physical cases. We summarize and conclude in section IV.

\section{FORMALISM}

The nucleon-meson interaction Lagrangian can be written as

$$
\begin{array}{r}
\mathcal{L}_{i n t}=g_{\sigma} \bar{\psi} \phi_{\sigma} \psi+g_{a_{0}} \bar{\psi} \phi_{a_{0}, a} \tau^{a} \psi+g_{\omega N N} \bar{\psi} \gamma_{\mu} \psi \omega^{\mu}+ \\
g_{\rho}\left[\bar{\psi} \gamma_{\mu} \tau^{\alpha} \psi+\frac{\kappa_{\rho}}{2 m_{n}} \bar{\psi} \sigma_{\mu \nu} \tau^{\alpha} \partial^{\nu}\right] \rho_{\alpha}^{\mu}
\end{array}
$$

where $\psi, \phi_{\sigma}, \phi_{a_{0}}, \rho$ and $\omega$ correspond to nucleon, $\sigma, a_{0}, \rho$ and $\omega$ fields, and $\tau$ s are Pauli matrices. The values used for the coupling parameters are obtained from Ref. 23]. From now on we use s and v to denote scalar and vector mesons, i.e. $\mathrm{s}=\sigma, a_{0}$ and $\mathrm{v}=\omega, \rho$. It is understood that $\kappa_{\omega}=0.0$.

The polarization vector through which the scalar meson couples to the vector meson via the $\mathrm{n}-\mathrm{n}$ loop is given by

$$
\Pi_{\mu}\left(q_{0},|\vec{q}|\right)=2 i g_{s} g_{v} \int \frac{d^{4} k}{(2 \pi)^{4}} \operatorname{Tr}\left[G(k) \Gamma_{\mu} G(k+q)\right],
$$

where 2 is an isospin factor and the vertex for v-nn coupling is:

$$
\Gamma_{\mu}=\gamma_{\mu}-\frac{\kappa_{v}}{2 m_{n}} \sigma_{\mu \nu} q^{\nu}
$$

$G(k)$ is the in-medium nucleon propagator given by 24

$$
G(k)=G_{F}(k)+G_{T}(k)
$$

with

$$
G_{F}(k)=\frac{\left(\not k+m_{n}^{*}\right)}{k^{2}-m_{n}^{* 2}+i \epsilon}
$$

and

$$
G_{T}(k)=\left(\not k+m_{n}^{*}\right) \frac{i \pi}{E_{k}}\left[\frac{\delta\left(k_{0}-E_{k}\right)}{e^{\beta\left(E_{k}^{*}-\nu\right)}+1}+\frac{\delta\left(k_{0}+E_{k}\right)}{e^{\beta\left(E_{k}^{*}+\nu\right)}+1}\right]
$$

where $E_{k}^{*}=\sqrt{\mathbf{k}^{2}+m_{n}^{* 2}}$ and $\nu=\mu-\frac{g_{v}^{2} \rho_{B}}{m_{v}^{2}}$ is the effective chemical potential. In Eq. (可), the subscripts $F$ and $T$ refer to the free and temperature-dependent part of the propagator. $G_{F}$ is the free Dirac propagator with the nucleon mass replaced by its effective value while the second term $\left(G_{T}\right)$ includes the Fermi distribution of the nucleon assuming thermal equilibrium at temperature $\mathrm{T}$. The delta function guarantees that on-mass-shell levels are occupied by the appropriate distribution functions. $m_{n}^{*}$ denotes the effective nucleon mass evaluated at the mean field level [24]. It's value is given by the equation:

$$
m_{n}^{*}=m_{n}-\frac{g_{\sigma}^{2}}{m_{\sigma}^{2}} \frac{\gamma}{(2 \pi)^{3}} \int d^{3} k \frac{m_{n}^{*}}{\left(\mathbf{k}^{2}+m_{n}^{* 2}\right)^{1 / 2}}\left[n_{k}(T)+\bar{n}_{k}(T)\right]
$$

where $n_{k}(T)=\frac{1}{e^{\beta\left(E_{k}^{*}-\nu\right)}+1}, \quad \bar{n}_{k}(T)=\frac{1}{e^{\beta\left(E_{k}^{*}+\nu\right)}+1}$ are the nucleon and anti-nucleon thermal distributions. It should be mentioned that in asymmetric nuclear matter the mean field generated by the $a_{0}$ meson would modify the neutron and proton mass differently. In the present case this effect vanishes.

In this notation the nucleon density is written as 


$$
\rho_{B}=\frac{\gamma}{(2 \pi)^{3}} \int d^{3} k\left[n_{k}(T)-\bar{n}_{k}(T)\right] .
$$

Now we have a set of two coupled equations, Eqs. (8 and 9) and two unknowns: the effective nucleon mass $m_{n}^{*}$ and the effective chemical potential $\nu$. One looks for the self-consistent solutions of these two coupled equations in order to find $m_{n}^{*}$ and $\nu$. The results are shown in Fig. 1 for two different nuclear densities. At a fixed density the effective nucleon mass increases and then again drops with temperature (T). This is consistent with Ref. [24]. It might be mentioned here that we do not include resonances in the present work.

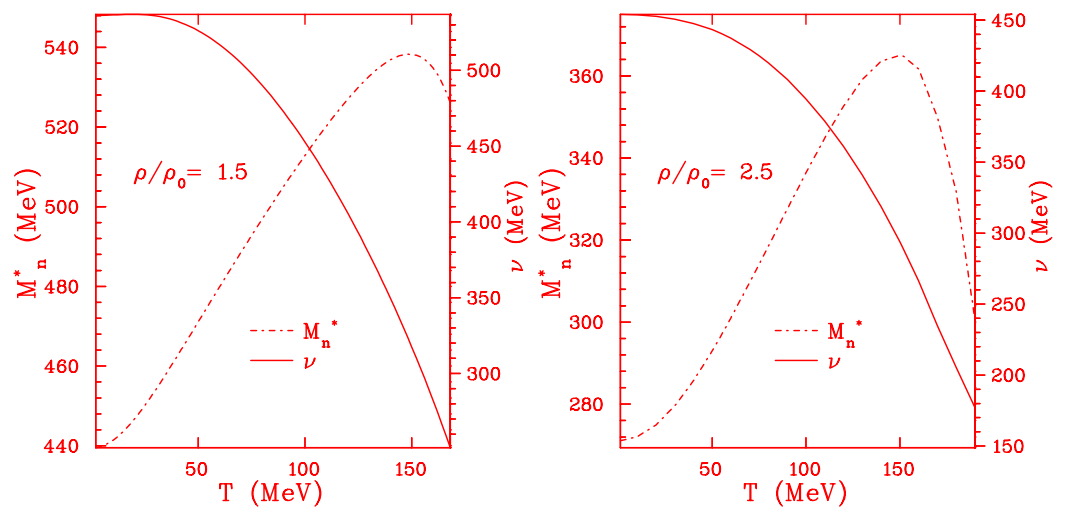

FIG. 1. The in-medium nucleon mass and chemical potentialas function of temperature for two baryonic densities.

Even though Eq. (3) involves Green's functions having both vacuum and temperature dependent part, the vacuum piece for $\Pi_{\mu}$, as expected vanishes on account of Lorentz symmetry. Hence only the thermal part contributes to the mixed-polarization function and it has a natural cut-off provided by the thermal distribution functions. So, interestingly, the diagrams involving mixing of different spin states in matter is free from ultra-violet divergences. It should also be noted that the argument of $\Pi_{\mu}$ involves $q_{0}$ and $|q|$ indicating Lorentz symmetry breaking in matter. In general, it could be a function of $\vec{q}$ but for an isotropic medium it can only depend on the magnitude of the three-momentum.

Returning to Eq. (3), after the evaluation of the trace and a little algebra, $\Pi_{\mu}$ could be cast into a suggestive form:

$$
\Pi_{\mu}\left(q_{0},|q|\right)=\frac{g_{s} g_{v}}{\pi^{3}} 2 q^{2}\left(2 m_{n}^{*}-\frac{\kappa q^{2}}{2 m_{n}}\right) \int_{0}^{\infty} \frac{d^{3} k}{E_{k}^{*}} \sum_{k_{0}= \pm E_{k}^{*}} \frac{k_{\mu}-\frac{q_{\mu}}{q^{2}}(k \cdot q)}{q^{4}-4(k \cdot q)^{2}} \frac{1}{e^{\beta\left(E_{k}^{*}+\operatorname{sgn}\left(k_{0}\right) \nu\right)}+1} .
$$

This immediately leads to two conclusions. First, it respects the current conservation condition $q^{\mu} \Pi_{\mu}=0=\Pi_{\nu} q^{\nu}$. Secondly, there are only two components which survive after the integration over azimuthal angle. In fact this guarantees that it is only the longitudinal component of the $\rho$ meson which couples to the scalar meson while the transverse mode remains intact. Furthermore, current conservation implies that out of the two non-zero components of $\Pi_{\mu}$, only one is independent. After performing the angular integration $\Pi_{0}$ takes the form:

$$
\begin{aligned}
\Pi_{0}=\frac{g_{s} g_{v}}{8 \pi^{2}|\vec{q}|}\left(2 m_{n}^{*}-\frac{\kappa q^{2}}{2 m_{n}}\right) \int_{0}^{\infty} \frac{k d k}{E_{k}^{*}} & \left\{2 E_{k}^{*} \ln \left[\frac{\left(q^{2}+2 k|\vec{q}|\right)^{2}-4 E_{k}^{* 2} E_{q}^{* 2}}{\left(q^{2}-2 k|\vec{q}|\right)^{2}-4 E_{k}^{* 2} E_{q}^{* 2}}\right]-q_{0} \ln \left[\frac{q^{4}-4\left(E_{k}^{*} E_{q}^{*}-4 k|\vec{q}|\right)^{2}}{q^{4}-4\left(E_{k}^{*} E_{q}^{*}+4 k|\vec{q}|\right)^{2}}\right]\right\} \\
\times & {\left[\frac{1}{e^{\beta\left(E_{k}^{*}-\nu\right)}+1}-\frac{1}{e^{\beta\left(E_{k}^{*}+\nu\right)}+1}\right] . }
\end{aligned}
$$

From Eq. (11) it is clear that the mixing amplitude vanishes for chemical potential $\nu=0$. This is expected, as such mixing between $\sigma$ and $\omega$ (or $\rho$ - $a_{0}$ ) opens up new channels which violate G-parity conservation. This can only happen when the ground state is not invariant under charge conjugation as is the case in nuclear matter with finite nucleon chemical potential. In fact, isospin eigenstate $\omega$, which (ignoring small mixing with $\rho$ induced by the quark mass difference and electromagnetic effects ) in free space cannot decay into two pions, in a thermal bath through its coupling with nucleon-antinucleon (or hole) excitation can decay into a pion pair with intermediate $\sigma$ meson [18]. The same argument holds true for $\rho$ which in matter can decay into $\pi-\eta$ [5].

Note that the mixing amplitude vanishes at $|q|=0$. Physically this corresponds to the situation when there is no way for the vector particle to distinguish between longitudinal and transverse direction and therefore rotational 
invariance forces them to be degenerate forbidding mixing of different spin states in this limit. On the other hand, it has also been observed that at very high momenta $\Pi_{0}$ falls off. This corresponds to the situation when collective effects, which basically related to the long wave length oscillations, die off with high momenta. This is a well-known phenomenon in many-body physics.

\section{A. Isoscalar channel : $\sigma-\omega$ mixing}

As has been mentioned before that $\sigma-\omega$ mixing has been studied previously in the context of dilepton production for $\pi-\pi$ annihilation in the s-wave channel in Ref. [18,19]. The thermal production rates of the dilepton from this channel however not been estimated before. In order to calculate the rate of dilepton production, we first calculate the cross-section for the process $\pi+\pi \rightarrow \sigma \rightarrow \omega \rightarrow e^{+}+e^{-}$. The Feynman diagram for the relevant process are shown in fig 2 .

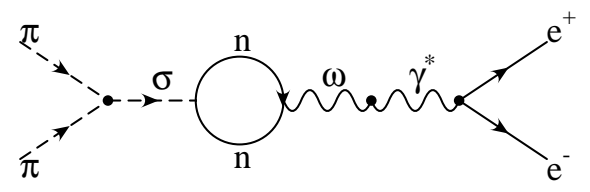

FIG. 2. The Feynman diagram for the process $\pi+\pi \rightarrow e^{+}+e^{-}$.

The interaction Lagrangian for the $\sigma-\pi \pi$ vertex is given by

$$
\mathcal{L}=\frac{1}{2} g_{\sigma \pi \pi} m_{\pi} \phi_{\sigma} \vec{\phi}_{\pi} \cdot \vec{\phi}_{\pi}
$$

The cross-section can easily be evaluated to be :

$$
\sigma_{\pi \pi \rightarrow e^{+} e^{-}}=\frac{2 \pi \alpha^{2}}{3 q_{z}^{2} M} \frac{\left(g_{\sigma \pi \pi} m_{\pi}\right)^{2}}{g_{\omega}^{2}} \frac{F_{\omega}\left(M^{2}\right) G_{\sigma}\left(M^{2}\right)}{\mathbf{k}_{\sigma}}\left|\Pi_{0}\right|^{2}
$$

where, $\Pi_{0}$ is given by Eq. (11), and

$$
\begin{gathered}
F_{\omega}\left(M^{2}\right)=\frac{m_{\omega}^{4}}{\left(M^{2}-m_{\omega}^{2}\right)^{2}+m_{\omega}^{2} \Gamma_{\omega}^{2}}, \quad G_{\sigma}\left(M^{2}\right)=\frac{1}{\left(M^{2}-m_{\sigma}^{2}\right)^{2}+m_{\sigma}^{2} \Gamma_{\sigma}^{2}(M)}, \\
\mathbf{k}_{\sigma}=\frac{\sqrt{\left(M^{2}-4 m_{\pi}^{2}\right)}}{2} .
\end{gathered}
$$

For $\omega$ we used a constant width of $9.5 \mathrm{MeV}$ and for the sigma meson we adopt the same mass and width as Ref. [19]. Recent discussion on this issue may be found also in Ref. [25,26]. We also have investigated the effects of form factors by considering the same form as in Ref. [18]: those are normalized to one for on-shell particles. Effects found are small as far as the dilepton yields are concerned.

\section{B. Isovector channel : $\rho-a_{0}$ mixing}

Quite similar to $\sigma-\omega$ mixing $\rho$ - $a_{0}$ mixing opens up a new channel for dilepton production viz. $\pi+\eta \rightarrow e^{+}+e^{-}$in dense nuclear matter through n-n excitations. The Feynman diagram of the process is shown in Fig. 3 .

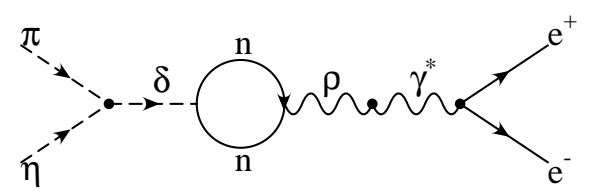

FIG. 3. The Feynman diagram for the process $\pi+\eta \rightarrow e^{+}+e^{-}$. 
To describe the $\pi a_{0} \eta$ vertex we use

$$
\mathcal{L}_{a_{0} \pi \eta}=f_{a_{0} \pi \eta} \frac{m_{a_{0}}^{2}-m_{\eta}^{2}}{m_{\pi}} \phi_{\eta} \vec{\phi}_{\pi} \cdot \vec{\phi}_{a_{0}} .
$$

For later convenience we define $g_{\pi a_{0} \eta}=f_{a_{0} \pi \eta}\left(m_{a_{0}}^{2}-m_{\eta}^{2}\right) / m_{\pi}$.

We should mention here that there is an uncertainty involved with the coupling parameter $f_{a_{0} \pi \eta}$ as discussed in Refs. [27,28. This arises from the fact that $a_{0}$ lies close to the opening of the $K \bar{K}$ channel leading to a cusp-like behavior in the resonant amplitude, therefore a naive Breit-Wigner form for the decay width is inadequate. Furthermore, as mentioned before, there is also uncertainty involved with the $a_{0} \mathrm{NN}$ coupling which renders the precise extraction of $a_{0}-\pi-\eta$ coupling even more difficult [27]. We take $f_{a_{0} \eta \pi}=0.44$ [5] which gives $\Gamma_{a_{0} \rightarrow \pi \eta}\left(m_{a_{0}}\right)=59 \mathrm{MeV}$, while the experimental vacuum width of $a_{0}$ is between $50-100 \mathrm{MeV}$ [28]. This choice is thus a conservative one.

As before, the cross-section involving mixing in the isovector channel can be written in terms of momentum and temperature dependent mixing amplitude:

$$
\sigma_{\pi \eta \rightarrow e^{+} e^{-}}=\frac{2 \pi \alpha^{2}}{3 q_{z}^{2} M} \frac{g_{a_{0} \pi \eta}^{2}}{g_{\rho}^{2}} \frac{F_{\rho}\left(M^{2}\right) G_{a_{0}}\left(M^{2}\right)}{\mathbf{k}_{a_{0}}}\left|\Pi_{0}\right|^{2}
$$

where

$$
F_{\rho}\left(M^{2}\right)=\frac{m_{\rho}^{4}}{\left(M^{2}-m_{\rho}^{2}\right)^{2}+m_{\rho}^{2} \Gamma_{\rho}^{2}(M)} \quad G_{a_{0}}\left(M^{2}\right)=\frac{1}{\left(M^{2}-m_{a_{0}}^{2}\right)^{2}+m_{a_{0}}^{2} \Gamma_{a_{0}}^{2}(M)}
$$

and the decay widths of the $a_{0}$ and $\rho$ mesons:

$$
\begin{gathered}
\Gamma_{a_{0}}(M)=\frac{f_{a_{0} \pi \eta}^{2}}{8 \pi}\left(\frac{m_{a_{0}}^{2}-m_{\eta}^{2}}{m_{\pi}}\right)^{2} \frac{\mathbf{k}_{a_{0}}}{M^{2}}, \quad \Gamma_{\rho}(M)=\frac{g_{\rho \pi \pi}^{2}}{6 \pi} \frac{\left(\frac{M^{2}}{4}-m_{\pi}^{2}\right)^{\frac{3}{2}}}{M^{2}}, \\
\mathbf{k}_{a_{0}}=\frac{\sqrt{\left(M^{2}-\left(m_{\pi}+m_{\eta}\right)^{2}\right)\left(M^{2}-\left(m_{\pi}-m_{\eta}\right)^{2}\right)}}{2 M} .
\end{gathered}
$$

As was the case for the $\omega$, we choose the mass of the $a_{0}$ according to Ref. [28].

\section{DILEPTON PRODUCTION RATES}

Once we know the relevant cross-section for the dilepton channel, the thermal production rate of the lepton pairs (number of reactions per unit time, per unit volume $R_{12}^{e^{+} e^{-}}=d N_{e^{+} e^{-} \text {pairs }} / d^{4} x$ ) can be estimated in the independent particle approximation of kinetic theory 15$]$ :

$$
\frac{d R_{12}^{e^{+} e^{-}}}{d M^{2}}=\int \frac{d^{3} k_{1}}{(2 \pi)^{3}} f_{1}\left(\mathbf{k}_{1}\right) \int \frac{d^{3} k_{2}}{(2 \pi)^{3}} f_{2}\left(\mathbf{k}_{2}\right) \frac{d \sigma_{12}^{e^{+} e^{-}}}{d M^{2}}\left(s, M^{2}\right) v_{r e l}
$$

where $f_{1}, f_{2}$ are the thermal distributions of the 1,2 species and $v_{r e l}=\frac{\lambda^{1 / 2}\left(s, m_{1}^{2}, m_{2}^{2}\right) \sqrt{s}}{2 E_{1} E_{2}}$ with $\lambda(x, y, z)=x^{2}+y^{2}+$ $z^{2}-2 x y-2 x z-2 y z$ is the triangle function. This could be cast into the form:

$$
\begin{gathered}
\frac{d R_{12}^{e^{+} e^{-}}}{d M^{2}}=\frac{\lambda\left(M^{2}, m_{1}^{2}, m_{2}^{2}\right)}{2} \Phi(s) \\
\Phi(s)=\int \prod_{i=1,2} \frac{d^{3} p_{i} f_{i}\left(E_{i}\right)}{(2 \pi)^{3} E_{i}} \delta\left[s-\left(p_{1}+p_{2}\right)^{2}\right] \sigma_{12}^{e^{+} e^{-}}\left(E 1, E 2, M^{2}\right)
\end{gathered}
$$

where $f_{i}\left(E_{i}\right)=\frac{1}{e^{\beta E_{i}}+1}$ are the distribution functions for $\pi$ and $\eta$ mesons assuming thermal equilibrium. A departure from the standard notation should be noticed here. Usually, $\Phi(s)$ represents only the phase space factor [30]. In our case the invariant mass cross-section depends also on energies through $\Pi_{0}$, we include that in the definition of $\Phi(s)$.

After performing the angular integrations we get

$$
\Phi(s)=\frac{1}{(2 \pi)^{4}} \int_{m_{1}}^{\infty} d E_{1} f_{1}\left(E_{1}\right) \int_{E_{2}^{-}}^{E_{2}^{+}} d E_{2} f_{2}\left(E_{2}\right) \sigma_{12}^{e^{+} e^{-}}\left(E 1, E 2, M^{2}\right)
$$

where $E_{2}^{ \pm}=\frac{1}{2 m_{1}^{2}}\left[E_{1}\left(s-m_{1}^{2}-m_{2}^{2}\right) \pm p_{1} \lambda^{1 / 2}\left(s, m_{1}^{2}, m_{2}^{2}\right)\right] . \Phi(s)$ is then calculated numerically. 


\section{A. Rates with $\sigma-\omega$ mixing}

First we concentrate on the isoscalar channel or in other words the effect of $\sigma$ - $\omega$ mixing on the dilepton production rate. As the mixing amplitude now involves the $\omega$ propagator we observe a sharp peak in the vicinity of the $\omega$ mass. Obviously this mixing amplitude depends on the density and the temperature. The effects are more pronounced in Fig. 4 where we have higher density. No such peak is observed for the $\sigma$ meson which is sufficiently broad. We also plot the rates for $\pi-\pi$ and $K-\bar{K}$ annihilation for comparison. The left panel shows the results at $\rho / \rho_{0}=1.5$ and the right panel shows the same for $\rho / \rho_{0}=2.5$.
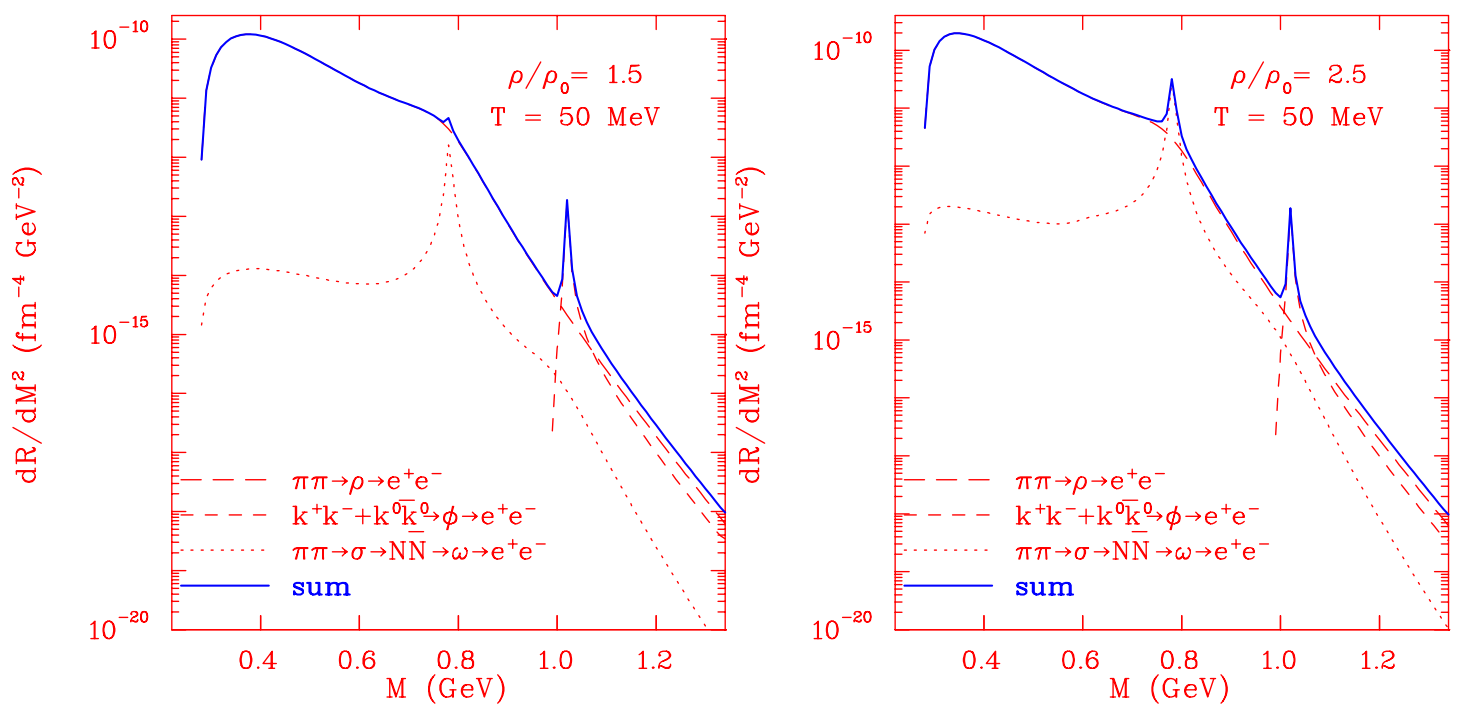

FIG. 4. Effect of $\sigma-\omega$ mixing on the dilepton production rate at $\mathrm{T}=50 \mathrm{MeV}$ for two different densities as mentioned in the legend.

Fig.5 shows the results for the isoscalar channel at a higher temperature. It is evident that at higher density the effect of mixing is also high.
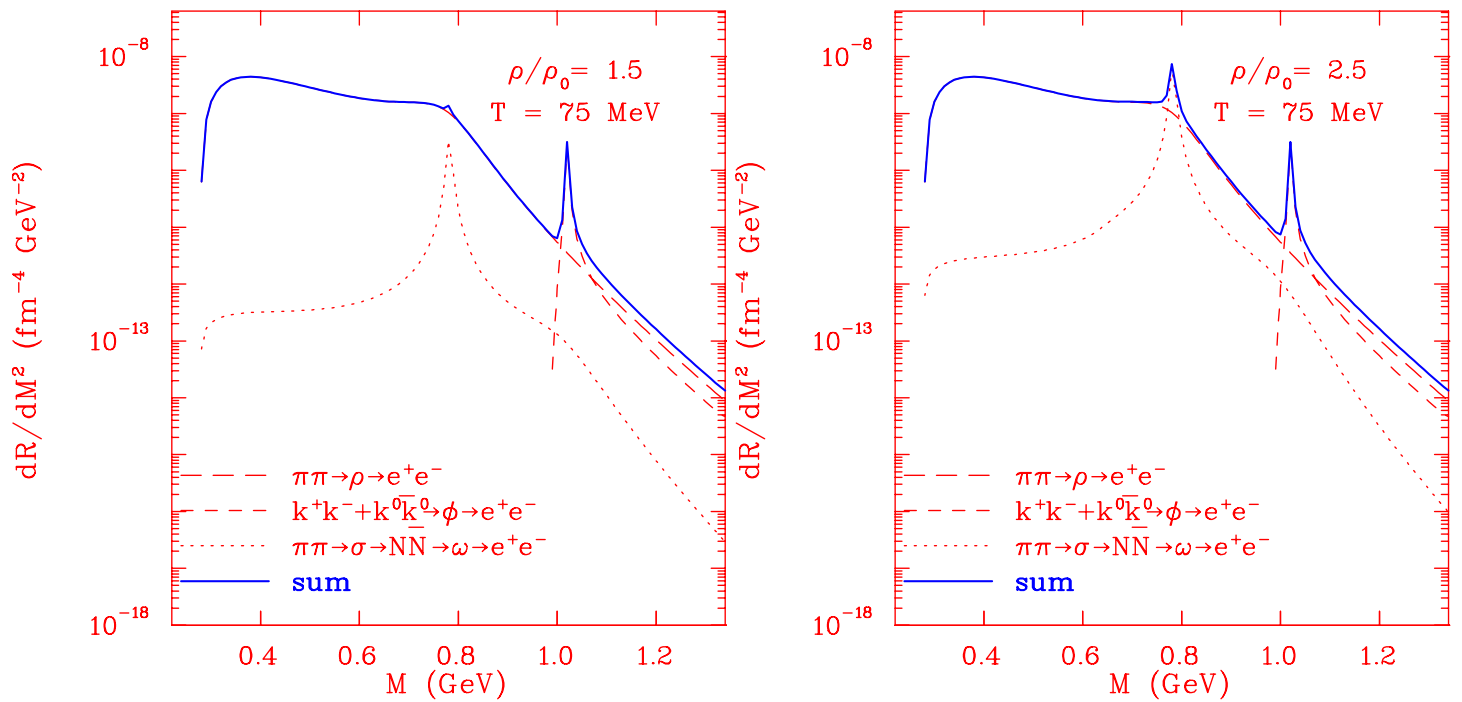

FIG. 5. Same as Fig. 4 but for $\mathrm{T}=75 \mathrm{MeV}$

It is to be noted that to calculate the dilepton rate we have used tree level propagator for $\omega$ and $\sigma$. In Ref. 18] it is argued that $\sigma-\omega$ mixing broadens the $\omega$ decay width substantially in nuclear matter at high density. This is understandable as now $\omega$ thorough nucleon-antinucleon (hole) excitations can decay into pion pairs as opposed to vacuum because of G-parity violation in matter. Therefore in matter this decay channel enjoys a larger phase space 
and becomes as broad as $\rho$ as its vacuum mass is almost degenerate with that of $\rho$ meson. But at the one loop level the diagonal elements of the mixed propagator would also reduce its mass (off-diagonal elements have little effect on the effective mass). Now if one considers the broadening induced by the off-diagonal element of the dressed propagator of the $\omega$ meson, then to be consistent one should also take the medium modified mass for the $\omega$ meson. It is well-known that in Walecka model $\omega$ meson mass also drops [29] which would again reduce the phase space. These two competing effects are not expected therefore to change the in-medium width of $\omega$ significantly. We, however, adopt a different point of view. Our focus here is to see the effect of mixing on the dilepton production and how it compares with $\pi-\pi$ annihilation. It should be noted that the contribution from $\sigma-\omega$ mixing does not change the resultant spectra much expect a small peak representing $\omega$ meson pole in the transition amplitude. However, we expect an $\omega$ peak in the dilepton spectra even in the absence of meson mixing. The situation, as we shall see, is different for the isovector mode. There the dominant contributions are distributed near the tail of the $\pi-\pi$ annihilation spectra ( $\rho$ spectral function). On the other hand if $\rho$ spectral function gets sufficiently broadened this effect might become more visible near the $\omega$ peak region where additional contributions would come from the mixing.

\section{B. Rates with $\rho-a_{0}$ mixing}

Next we discuss the effect of mixing in the isovector channel which involves $\rho-a_{0}$ mixing in Fig.6 (7) for $\mathrm{T}=50$ $\mathrm{MeV}(75 \mathrm{MeV})$. This induces an $a_{0}$ bump just below the $\phi$ peak. $\rho-a_{0}$ channel seems to be more interesting as it induces additional contribution beyond the tail of $\rho$ spectral function. One can easily observe from Fig 6 that even at density $\rho / \rho_{0}=1.5$ the contribution of the mixing wins over the $\pi-\pi$ annihilation rate in the vicinity of $\mathrm{M}=1.0$ $\mathrm{GeV}$. Naturally at higher density this goes up as evident from the right panel of Fig. 6 .
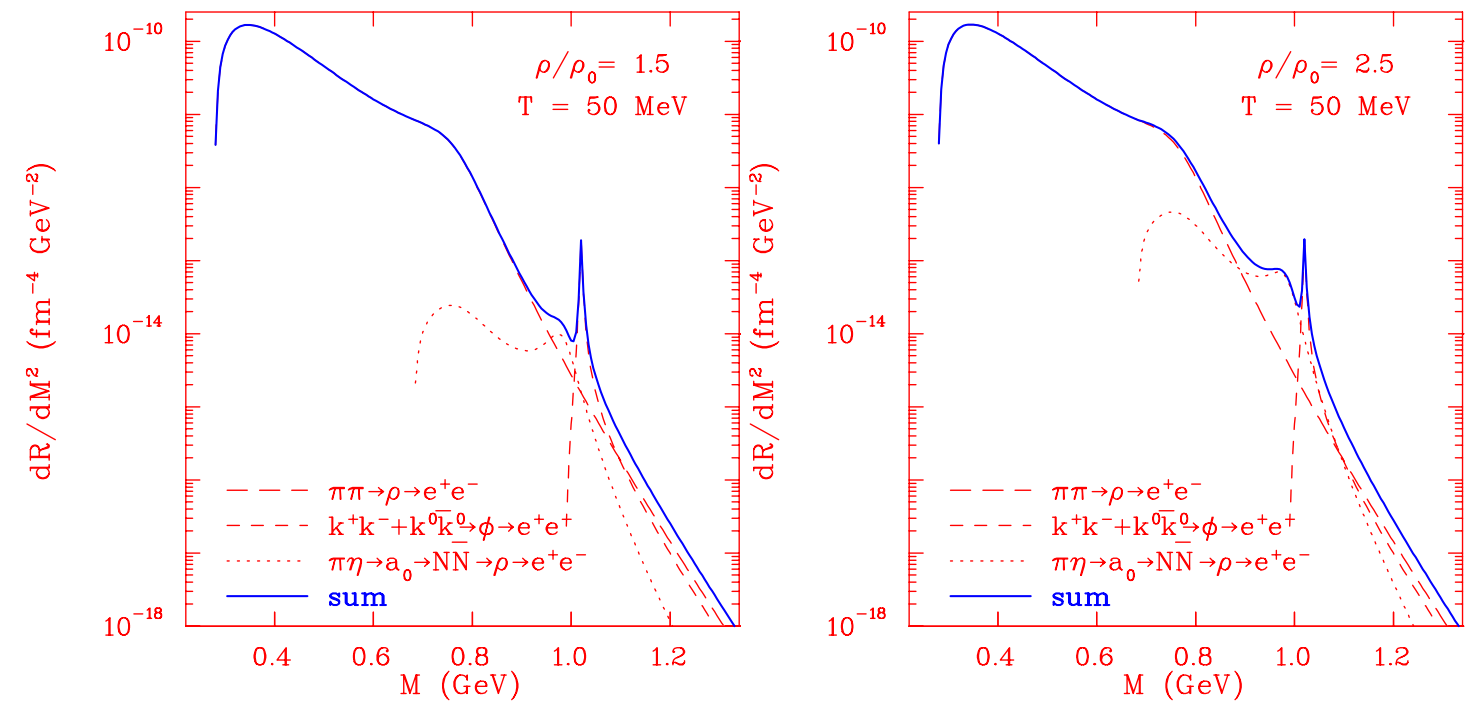

FIG. 6. Effect of mixing on dilepton production rate in the isovector channel involving $\rho-a_{0}$ mixing at $\mathrm{T}=50 \mathrm{MeV}$.

Interestingly enough the dilepton yield induced by the mixing in the isovector channel at higher temperature also seems to be significant as evident from the figures below. 

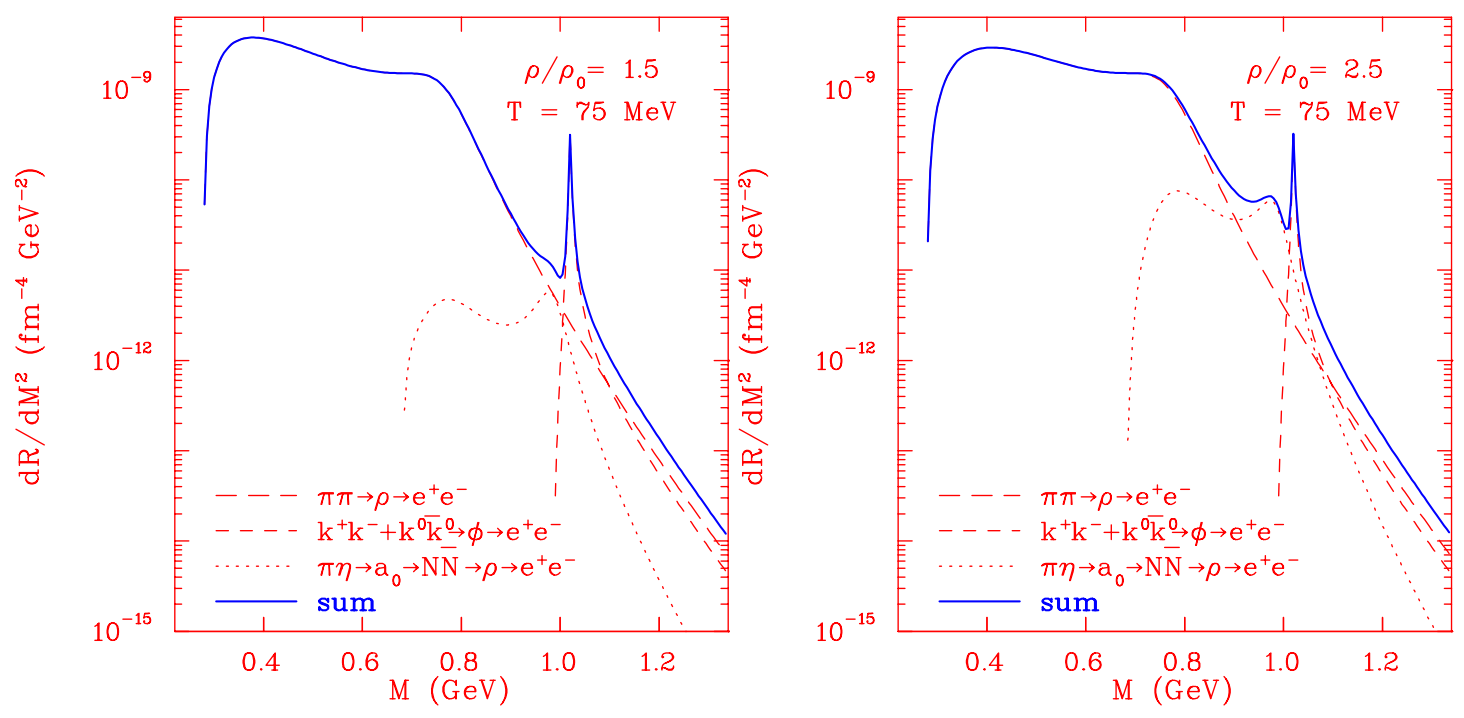

FIG. 7. Same as Fig. 6 but for $\mathrm{T}=75 \mathrm{MeV}$

Note that, unlike $\omega$, the broadening induced by the mixing on $\rho$ decay width has been found to be marginal. One can understand this physically. Mixing opens up a new channel for $\rho$ to decay into $\pi-\eta$. $\eta$ being quite heavy only the tail part of the $\rho$ can contribute. Therefore it does not enjoy much of phase space to decay into $\pi-\eta$. It does seem reasonable to conclude that $\rho$ - $a_{0}$ mixing provides more promising kinematical window in the invariant mass spectra to study exclusive in-medium effects.

\section{Rates with mixing both in isoscalar and isovector channels}

For completeness we show in Figs. 8 and 9 the total dilepton yield with the combined effects of mixing in both the isoscalar and isovector channels. It is important to see that rates induced by mixing are significant in certain windows of density and temperature.
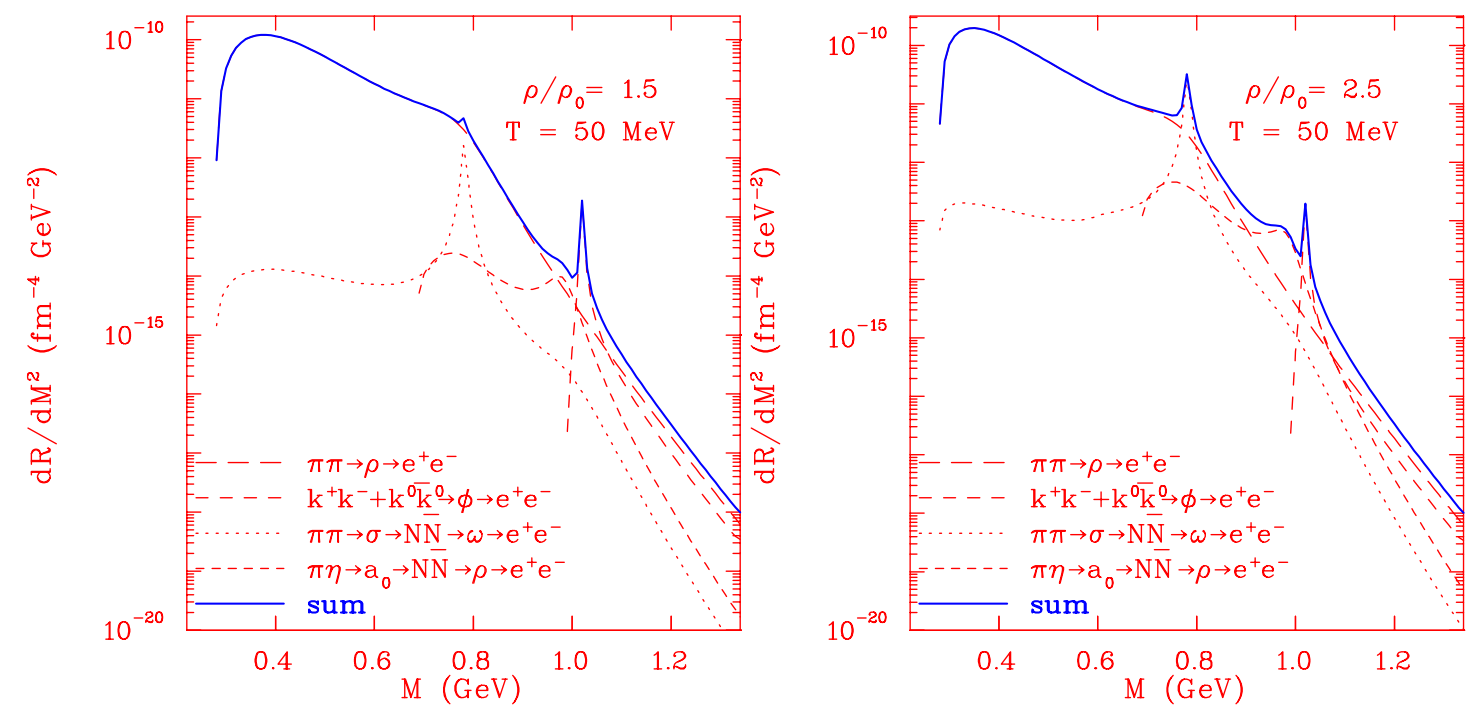

FIG. 8. Rates at $\mathrm{T}=50 \mathrm{MeV}$ with mixing in both isoscalar and isovector channels. 

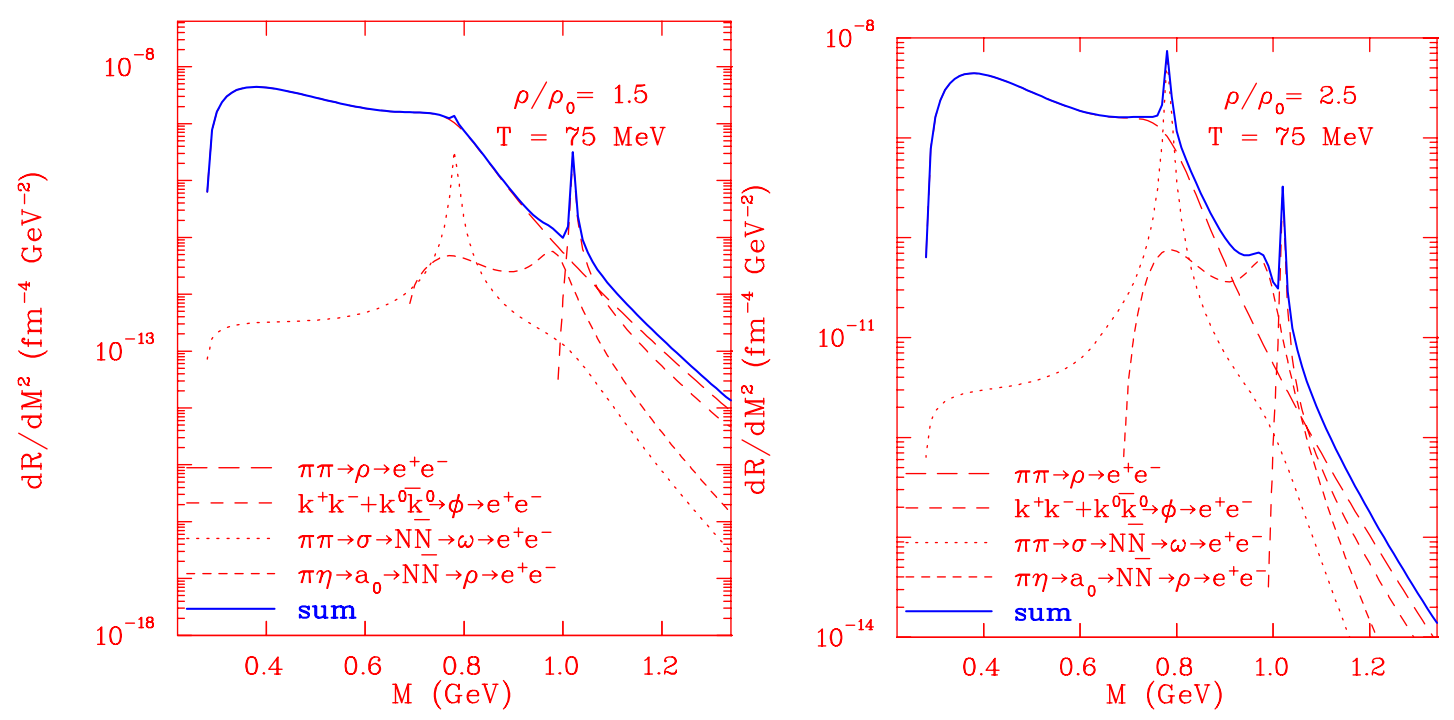

FIG. 9. Rates at $\mathrm{T}=75 \mathrm{MeV}$ with mixing in both isoscalar and isovector channels.

\section{SUMMARY AND CONCLUSION}

To conclude and summarise, we have estimated the dilepton production rates arising from scalar and vector meson mixing in different isospin channel involving nucleon -hole (or anti-nucleon) excitations as intermediate states. The rates have been compared against the $\pi-\pi$ annihilation in the $\mathrm{p}$-wave channel ( $\rho$-channel) and $K-\bar{K}$ annihilation. Higher yield of dileptons in the invariant mass region between the $\rho$ and the $\phi$ peaks are expected. The mixing in the isoscalar channel contributes mostly between the two-pion threshold and the $\omega$ peak but is small compared to the $\pi-\pi$ annihilation contribution. On the other hand, the isovector channel seems to provide a better probe as it contributes near the tail region of the two pion annihilation contribution. It should be stressed here that broadening or dropping $\rho$ meson mass would even favor our observation. In that case the $\pi-\pi$ background would be pushed more towards lower invariant mass region bringing the $a_{0}$ peak into a clearer relief. Finally, it is important to realize that mixing effects only appear in the longitudinal part of the vector meson spectral function and therefore there should be no effect of the mixing on the photon spectra which involves the transverse part of the vector meson polarization tensor.

The effects studied here are density driven and can therefore be observed in environments where one has high baryonic chemical potential and comparatively lower temperature. The most relevant experiments to observe this kind of medium-induced phenomenon are the ones to be performed at GSI using the HADES detector where the density might reach up to three times the normal nuclear matter density and temperature $\mathrm{T}=60-90 \mathrm{MeV}$ [31]. Ref. [32] also indicates a similar range of densities and temperatures at SIS/GSI energies. Those observations have guided our choice of the temperatures and densities used in this study.

The calculations performed here can, and will be improved upon. It is important to proceed cautiously. As a next step, it would be necessary to address this problem with the full machinery of finite temperature many-body theory. This means, among other things, the dressing of the propagators and the consistent inclusion of baryonic resonances. Also, the interaction zone in a heavy collision is but a transient medium and the dynamics will contribute to mask our signal to some extent. However, owing to the nature of the electromagnetic signal, radiation from the dense regions will emerge. In our approach those dynamics would impact on the height of the $a_{0}$ peak. Similarly, a realistic assessment of the initial states of the dilepton-producing channels should involve a realistic simulation. A transport treatment can therefore address the concerns raised above. Such investigations are under progress.

\section{ACKNOWLEDGMENTS}

This work was supported in part by the Natural Sciences and Engineering Research Council of Canada and in part by the Fonds FCAR of the Québec Government. 
[1] A. K. Dutt-Mazumder, B. Dutta-Roy, and A. Kundu, Phys. Lett. B399, 196 (1997).

[2] W. Broniowski and W. Florkowski, Phys. Lett. B440, 7 (1998).

[3] W. Broniowski and W. Florkowski, Eur. Phys. J. A7, 287 (2000).

[4] S. A. Chin, Ann. Phys. (N. Y.) 108, 301, (1977).

[5] O. Teodorescu, A.K. Dutt-Mazumder, C. Gale, Phys. Rev. C 61, 051901 (2000).

[6] G. E. Brown and M. Rho, Phys. Rev. Lett. 66, 2720 (1991).

[7] R. Rapp and J. Wambach, hep-ph/9909229, submitted to Adv. Nucl. Phys.

[8] See, for example, R. J. Porter et al., Nucl. Phys. A638, 499 (1998), and references therein.

[9] M. Masera for the HELIOS collaboration, Nucl. Phys. A590, 93c (1995).

[10] P. Wurm for the CERES collaboration, Nucl. Phys. A590, 103c (1995).

[11] P. Bordalo et al., Nucl.Phys. A661, 538 (1999).

[12] D. P. Morrison et al., Nucl. Phys. A638, 560 (1998).

[13] J. Friese et al., Prog. Part. Nucl. Phys. 42, 235 (1999).

[14] M. Kossov et al., TJNAF proposal No. PR-94-002 (1994).

[15] C. Gale and J. Kapusta, Phys. Rev. C 35, 2107 (1987); C. Gale and Lichard, Phys.Rev.D 49, 3338 (1994).

[16] K. L. Haglin and C. Gale, Nucl. Phys. B421, 613 (1994).

[17] R. Rapp and C. Gale, Phys. Rev. C 60, 024903 (1999).

[18] G. Wolf, B. Friman, and M. Soyeur, Nucl. Phys. A640, 129, (1998).

[19] K. Saito, K. Tsushima, A. W. Thomas, and A. G. Williams, Phys. Lett. B433, 243 (1998).

[20] S. Kubis and M. Kutschera, Phys.Lett. B399, 191 (1997).

[21] F. de Jong and H. Lenske, Phys. Rev. C 57, 3099 (1998).

[22] E. Oset and M. J. Vicente Vacas, nucl-th/0004030.

[23] R. Machleidt, Adv. Nucl. Phys. 19, 198 (1989).

[24] B. D. Serot and J. D. Walecka, Adv. Nucl. Phys. 16, 1 (1986).

[25] N. A. Tornquist and M. Roos, Phys. Rev. Lett. 76, 1575 (1996).

[26] M. Harada, F. Sannino and J. Schechter, Phys. Rev. D 54, 1991 (1996); Phys. Rev. Lett. 78, 1603 (1997).

[27] M. Kirchbach and L. Taitor, Nucl. Phys. A604, 385 (1996).

[28] Particle Data Group, Euro. Phys. J. C, Vol. 3, 1 (1998).

[29] T. Hatsuda , H. Shiomi and H. Kuwabara, Prog. Theor. Phys. 95, 1009 (1996).

[30] P. Lichard, Phys. Rev. D 495812 (1994).

[31] J. Friese, Nucl.Phys. A654, 311 (1999); Prog. in Part. and Nucl. Phys. 42, 235 (1999).

[32] A. Devismes, Proc. Int. Workshop XXVIII on Gross Properties of Nuclei and Nuclear Excitations, Hirschegg, 2000. 